\title{
Disturbances Impact on Longevity of Grass Seeds, Semi-Arid South African Rangeland
}

\author{
H. A. Snyman \\ Author is Professor, Department of Animal, Wildlife and Grassland Sciences, University of the Free State, Bloemfontein 9300, South Africa.
}

\begin{abstract}
The effect of plant and soil disturbances on seed density, species richness, and seed longevity of the soil seed bank was quantified for a semi-arid rangeland, over a 5-yr period (2002/2003-2006/2007 growing seasons). The different soil and plant treatments included fire, tillage (intended as a trampling surrogate), and blocked seed rain (simulating heavy grazing). These three experimental factors were combined in a factorial arrangement. Seed responses were evaluated in the soil seed bank before the new seed set, after the first seed production event, and after the second seed production event. Before disturbance (physical impact on the plant and soil), soil seed bank was dominated by early successional species: conversely, aboveground vegetation was mainly dominated by perennial grasses. After only $4 \mathrm{yr}$ of blocked seed rain, seedling emergence of Decreaser grass species ceased totally both in the field and seed bank, with lower effect on Increaser grass species. Emergence of both Decreaser and Increaser grass species decreased in the seed bank with tillage, whereas the opposite occurred in the field. By contrast, tillage increased the emergence of weeds in the seed bank. The decrease in emergence of Decreaser grass species in both seed bank and field was still evident $4 \mathrm{yr}$ after the rangeland was burnt. The grass species Themeda triandra was the most sensitive to fire in terms of seedling emergence. Blocked seed rain treatment significantly decreased $(P<0.05)$ species richness. Regardless of treatments applied, there was poor similarity between aboveground vegetation and the associated seed bank. Differences in the soil seed bank are likely to reflect manifested properties rather than short-term changes. Several characteristics of seed banks (species composition, seed abundance, and longevity) must be considered in order to understand the dynamics of plant communities following disturbances.
\end{abstract}

Key Words: blocked seed rain, fire, seedling emergence, species richness, tillage

\section{INTRODUCTION}

The soil seed bank plays an important role in the composition of different plant communities and thus in their conservation (López-Marińo et al. 2000; Shauhat and Siddiqui 2004). The composition of the seed bank depends on the production and composition of the present and previous communities (Harrington et al. 1984; Fenner 1985) as well as on the longevity of the seeds of each species under local conditions (Bekker et al. 1997; Thompson and Grime 1997). Vegetation studies in grazing systems are mostly restricted to the aboveground vegetation, and often ignore the role that soil seed banks could play in the restoration of degraded vegetation communities after disturbance (de Villiers et al. 2003; Solomon et al. 2006; Amaha Kassahun et al. 2009; Dereber et al. 2011).

The trampling and removal of vegetation by animals or fire have a significant impact on the number of seeds produced by a plant and released as seed rain (Page and Beeton 2000; Snyman 2005). According to Chang et al. (2001), regeneration strategies of plants are shaped by patterns of disturbance and stress. These act as selective forces over evolutionary time. The presence of seeds in disturbed habitats is determined by the relationship between the original plant assemblages, the

The research was partially funded by the National Research Foundation (NRF) South Africa.

Correspondence: H. A. Snyman, Dept of Animal, Wildlife and Grassland Sciences, University of the Free State, P0 Box 339, Bloemfontein 9300, South Africa. Email: Snymanha@ufs.ac.za

Manuscript received 18 August 2011; manuscript accepted 5 August 2012.

(c) 2013 The Society for Range Management amount of propagule production, and the capacity to build up seed reserves in the soil (Kinucan and Smeins 1992; Chang et al. 2001). In rangeland management, it is critical to establish how far an ecosystem can deviate from a reference state before being at risk to cross a threshold into an alternative stable state from which it is unable to revert without active intervention (Briske et al. 2008; Dreber and Esler 2011). Once the seed bank changes, the resulting community structure will be different and therefore seed banks have the potential to represent a threshold.

In relatively undisturbed rangelands, the role played by seedling recruitment from the persistent seed bank in vegetation composition changes, and its importance compared with recent seed rain is not always clear (Bullock et al. 1994; Page and Harrington 2009). Edwards and Crawley (1999) argued that the extent of seedling recruitment from the seed bank and seed rain is not only significant to our general understanding of how plant species richness is maintained, but also has applied significance for attempts to restore species richness in speciespoor grasslands (Page et al. 2006; Snyman 2009). Rangelands have a large, persistent seed bank, often with a species composition that does not resemble the aboveground vegetation (Thompson and Grime 1997; Amaha Kassahun et al. 2009), and it is well documented that these seeds can dictate the successional trends that occur following large-scale disturbances (Bekker et al. 1997; Edwards and Crawley 1999).

Worldwide, rangelands are subject to active management and these practices are based on a variety of criteria and constraints (Snyman 2009). Periodically, grazing and burning is commonly used to reduce competitive effects among plant species (Savory and Parsons 1980; Heitschmidt and Walker 
1983; Bakker 1989; Snyman 2004). Rangeland degradation through heavy grazing can decrease species richness both in the seed bank and seedling establishment in the field (Snyman 2004), while fire can over the short-term stimulate seedling density from the seed bank (Snyman 2005). Large parts of the semi-arid rangelands of southern Africa are characterized by large-scale accidental or human-caused runaway fires, driven by August winds (Snyman 2004). Fire is seldom isolated from its association with grazing and trampling. When the production potential of these rangelands is overestimated after burning, the resulting grazing would cause a decline in rangeland condition through species selection and trampling (Snyman 2006). Accidental, runaway fires and trampling will not only have a short-term influence on ecosystem functioning but may also have a major residual effect on the next growing season, depending on subsequent climatic conditions and postfire management (Snyman 2005). Heavy grazing is considered the most important cause of rangeland degradation in South Africa. The ecologically sensitive semi-arid rangeland areas are increasingly subjected to severe grazing pressures, which cause their rapid deterioration (van der Westhuizen 1999). For example, in some communal grazing areas, plants are not allowed to seed due to a system of continuous heavy grazing (Solomon et al. 2006; Rutherford and Powrie 2011). The understanding of the function and dynamics of seed banks has become a great challenge to ecologists working in plant communities, as this understanding is necessary to determine the role of the seed bank in ecosystem functioning and to improve the integrated management of ecosystems (Luzuriaga et al. 2007; Snyman 2009; Dreber 2011).

It is important to know the degree to which species in a system depend on specific forms of disturbance or whether various types of disturbance have equivalent effects on the soil seed bank (Bekker et al. 1997; Page et al. 2006; Ma et al. 2010). Whereas some authors have argued that prescribed burning should be the preferred form of rangeland management (Everson 1999; Trollope 1999), others have suggested that a variety of forms of disturbance can have equivalent effects (Collins et al. 1998; Jutila and Grace 2002). The potentially adverse effects of disturbance, particularly when intense and/or frequent, must also be given careful consideration (Jutila and Grace 2002; Laterra et al. 2006). Unfortunately, there are only few studies about the regenerative potential of seed banks (Luzuriaga et al. 2007), the longevity of the seeds of each species under specific climatic conditions, and the quantification of seed rain in arid and semi-arid areas. In this study, it is hypothesized that with soil and plant disturbance (through fire, trampling, and heavy grazing), seedling emergence and species richness within different functional groups of species will decrease over time in both the seed bank and field. These effects will vary depending on the botanical composition of the aboveground vegetation and recurrence of the disturbance.

\section{MATERIALS AND METHODS}

\section{Site Description}

The field study was conducted at Bloemfontein (lat $28^{\circ} 50^{\prime} \mathrm{S}$, long $26^{\circ} 15^{\prime} \mathrm{E}$, altitude $1350 \mathrm{~m}$ above sea level), situated in the semi-arid region of South Africa. Rain falls almost exclusively during the summer (October to April), with a mean annual rainfall of $560 \mathrm{~mm}$ and 78 rainy days per year. Mean maximum monthly temperatures range from $17^{\circ} \mathrm{C}$ in July to $33^{\circ} \mathrm{C}$ in January, with a mean of 119 frost-days annually (ISCW 1998).

The study area is situated in the eastern Free State Sandy Grassland (vegetation type-GM4) described by Mucina and Rutherford (2006), situated in the Grassland Biome (Bredenkamp and van Rooyen 1996). The botanical composition and basal cover of the rangeland was typical of a well-managed commercial farm in the area and described in detail by Snyman (2000). The soil is a fine sandy loam of the Bloemdal form (Roodeplaat family, 3200 ; Soil Classification Working Group 1991). Clay content increases with soil depth from $10 \%$ in the A-horizon $(0-300 \mathrm{~mm})$, to $24 \%$ in the $\mathrm{B}_{2}$-horizon $(300-600$ $\mathrm{mm})$, and $42 \%$ in the $B_{2}$-horizon $(500-1200 \mathrm{~mm})$. Bulk densities were $1484 \mathrm{~kg} \cdot \mathrm{m}^{-3}$ for horizon $\mathrm{A}, 1563 \mathrm{~kg} \cdot \mathrm{m}^{-3}$ for horizon $\mathrm{B}_{1}$, and $1758 \mathrm{~kg} \cdot \mathrm{m}^{-3}$ for horizon $\mathrm{B}_{2}$, and their upper limits of the soil-water holding capacity were $69 \mathrm{~mm}, 73 \mathrm{~mm}$, and $82 \mathrm{~mm}$, respectively (Snyman 2000).

Grazing history of the study site before applying the treatments was an annually winter (August) harvest (stubble height, $30 \mathrm{~mm}$ ) over the previous $9 \mathrm{yr}$. The site was also protected from any grazing or fire. Winter burning is a common management practice in these areas.

\section{Treatments}

A $2 \times 2 \times 2$ factorial experiment was conducted on 24 plots of $10 \times 10 \mathrm{~m}$ each, with a buffer zone of $5 \mathrm{~m}$ around each plot. Experimental treatments were fire, tillage, and blocked seed rain. Treatments were assigned randomly to plots $(n=3$ per treatment). The treatment combination of seed rain, no tillage, and no fire can be seen as the control.

The fire treatment was a once-off burn (head fire; Trollope 1999) carried out on 30 August 2002 after the first spring rainfall. Burning was carried out in the morning with a light wind blowing and the grass fuel initially very dry. The mean height of the flames was $1.25 \mathrm{~m}$ once the fire was burning uniformly. If the environmental parameters obtained with this study namely aboveground phytomass production (1453 $\left.\mathrm{kg} \cdot \mathrm{ha}^{-1}\right)$, fuel-water content $(18 \%)$, wind speed $\left(2.44 \mathrm{~m} \cdot \mathrm{s}^{-1}\right)$, and relative humidity $(43 \%)$ were built into the fire behavior model of Trollope (1999), the predicted fire intensity is 1145 $\mathrm{KJ} \cdot \mathrm{s}^{-1} \cdot \mathrm{m}^{-1}$. Therefore, the fire intensity ranged between moderate and hot (Trollope and Potgieter 1985). The frequency distribution of maximum temperatures recorded at ground level, $10 \mathrm{~mm}$ beneath the soil surface, grass canopy height, and $1 \mathrm{~m}$ above the ground were $100^{\circ} \mathrm{C}, 6^{\circ} \mathrm{C}, 600^{\circ} \mathrm{C}$, and $400^{\circ} \mathrm{C}$, respectively. The measurement procedures for the different fire parameters are discussed in detail by Snyman (2006). To limit the fire to each burnt plot, the plants surrounding each plot were cut short and soaked before burning.

The tillage treatment attempted to imitate the trampling by stock (sheep). The soil between the tufts were loosened/tilled twice per year (end of September through spring and end of January through summer) by means of a roller with sharpened stakes/tines $(10 \mathrm{~mm}$ diameter) spaced $40 \mathrm{~mm}$ apart with a penetration of $5 \mathrm{~mm}$ into the soil. This disturbance was applied when the soil was wet. A rain shower of between $10 \mathrm{~mm}$ and 
$15 \mathrm{~mm}$ was taken as the norm to apply this disturbance action on the day following the rain.

In the last treatment (blocked seed rain), the seed culms were often removed by cutting as soon as the growing points began lengthening so that the seeds of the grasses never ripened. No seed production from the plants or seed rain took place and therefore suggests that seeds were recovered from the soil surface and/or from the soil seed bank (Bullock et al. 1994; Hodgkinson and Freudenberger 1997). This treatment was therefore mimicking heavy grazing and applied to determine the longevity of seeds in this semi-arid climate. In the control treatment, the grasses were allowed to seed and the seed to ripen and fall on the ground as seed rain.

\section{Data Collection in the Field}

This research was conducted over five growing seasons (2002/ 2003-2006/2007). To determine the botanical composition of the field before applying the treatments, a bridge-point apparatus to perform a pin-point analysis of vegetation composition (Walker 1970; Snyman and Fouché 1991) was used. Five hundred points (nearest plant) were recorded per plot. Floristic composition was summarized as the percentage contribution of each species based on total points per plot. Rangeland condition was determined according to the degradation gradient technique of van der Westhuizen et al. (1999). The rangeland species were classified considering their desirability to animals in terms of grazing value (dry-matter production, palatability, nutritive value, whether perennial or annual, and grazing resistance; van der Westhuizen et al. 1999) as well as their ecological status (Decreaser or Increaser grass and shrub species), as defined by Hardy et al. (1999), were taken into consideration. Decreaser species were defined as those species that predominate in good condition rangeland but whose abundance declines when rangeland condition deteriorates through over- or underuse. Increaser species are those species not abundant in rangeland in good condition but whose abundance increases when rangeland is underused (i.e., as succession proceeds beyond the fire-grazing climax stage) or overused (Hardy et al. 1999). Classification of subtypes of Increaser species are explained in Table 1. This species classification into different ecological groups was chosen because it gives a good indication, not only of rangeland condition, but also the trend of botanical composition change. A weed was defined as a nongrass, which is considered undesirable.

Seedling emergence was registered in the field. This was accomplished by randomly distributing 10 quadrates $(0.5 \times 0.5$ $\mathrm{m}$ each) per treatment. Unfortunately, the survival of the identified seedlings was not monitored: only their emergence.

\section{Greenhouse Study}

Seed bank characteristics were determined under a controlled greenhouse study. Soil samples were collected randomly in eight blocks $(0.5 \times 0.5 \mathrm{~m}$ each $)$ annually in every treatment to a depth of $50 \mathrm{~mm}$ at the end of spring, summer, and autumn in the field. Field samples were collected into separate paper bags for immediate transport to the greenhouse for processing within $10 \mathrm{~min}$ of collection. In the greenhouse, soil samples were spread evenly in plastic containers $(0.5 \times 0.5 \mathrm{~m})$ contain- ing a 100-mm deep layer of Hygrotech growth medium (Canadian peat, polystyrene vermiculite, and mono-ammonium phosphate). To measure the extent of contamination, eight additional plastic containers filled with autoclave-sterilized soil $\left(90^{\circ} \mathrm{C}\right.$ for $1 \mathrm{~h}$, repeated three times over a week) were included with each set of soil samples. Seedling plastic containers were placed at random in the greenhouse. Containers were handwatered daily, after which the seedlings were identified and counted daily over a 2-mo period. All identifiable seedlings were removed. Seedlings that could not be identified after 2 mo were potted individually and grown until identification could be made. The soil medium ensured that the plants that germinated could reach a stage where they could be identified before dying down. Respective day and night temperatures of $25-30^{\circ} \mathrm{C}$ and $15-18^{\circ} \mathrm{C}$ were maintained in the greenhouse to simulate rangeland conditions.

The seed bank for determining seed density was annually investigated at the beginning of October (spring, before the new seed set), at the beginning of January (summer, after the first seed production event), and at the beginning of April (autumn, after the second seed production event). The phonological pattern of the vegetation in the study area is characterized by these two seed setting periods every season under normal rainfall conditions. Dreber (2011) mentioned the importance of considering seasonal variability in the availability of readily germinable seeds.

\section{Data Analysis}

A three-way factorial ANOVA at 95\% confidence level was performed (with three replications) to determine any significant differences between the three treatment combinations (fire, tillage, and blocked seed rain), where all interactions among treatments were included (Winer 1974). Species frequency data in the soil seed bank and field were collected as repeated measures, and analyses were conducted using SAS (Dos program, version 8.1, SAS 2001). Analysis of year effects, regarded as in situ treatments, was also conducted in SAS (SAS 2001). The ANOVA analysis for both the soil seed bank and field were univariate for each variable.

Seedling density in both the field and soil seed bank was also analyzed by separate ANOVAs to quantify differences within seasons. Tukey's procedure for comparison of means was applied for species composition and seedling density. The Number Cruncher Statistical System (2000) software package (Hintze 1997) was used for statistical analyses. Species richness was considered as the total number of grass species in seed banks or field occurring within all soil samples or quadrates for each treatment. Fisher's method (Fisher 1949) was used in determining the least significant difference (LSD) for species richness and seedling density, with $P<0.05$ considered significant.

Motyka's similarity index (Mueller-Dombois and Ellenberg 1974) was used to make comparisons between seed bank and field vegetation: similarity index $(\%)=2 c /(a+b) \times 100 \%$, where $\mathrm{c}$ is the number of species common to both samples, and $\mathrm{a}$ and $\mathrm{b}$ are the number of all species in sample $\mathrm{A}$ and all species in sample B, respectively. A permutation ANOVA, which can use Motyka's values, was conducted to test the pair differences (Anderson 2001). 
Table 1. Relative species frequency (\%) for the vegetation in the field (2002/2003) and of the survived seedlings emerging from the soil samples collected from the rangeland for different treatments, which were germinating in the greenhouse. Seed bank soil collected in autumn for the 2002/2003 and 2005/2006 growing seasons. Multiple comparisons among treatment means are apparently performed only for totals. No Decreaser species occurred for blocked seed rain for the $2005 / 2006$ season and therefore the gaps. Within a line for a season, different first letters indicate significant $(P<0.05)$ differences among blocked seed rain treatments within each fire treatment; different second letters indicate significant $(P<0.05)$ differences among tillage treatments within each fire treatment, and different third letters indicate significant $(P<0.05)$ differences between fire treatments within a blocked seed rain treatment, based on the Tukey test. Increaser I species are those species whose abundance increases when rangeland is underused and Increaser II species whose abundance increases when rangeland is overused (Increaser Ila, b, and c: increase in abundance with moderate, severe, and extremely severe overused, respectively).

\begin{tabular}{|c|c|c|c|c|c|c|c|c|c|}
\hline \multirow{4}{*}{$\begin{array}{l}\text { Species (ecological } \\
\text { status) }\end{array}$} & \multirow{4}{*}{$\begin{array}{c}\text { Field } \\
\text { (2002/2003 } \\
\text { growing } \\
\text { season) }\end{array}$} & \multicolumn{8}{|c|}{ Seedbank (2002/2003 growing season) } \\
\hline & & \multicolumn{4}{|c|}{ Seed rain } & \multicolumn{4}{|c|}{ Blocked seed rain } \\
\hline & & \multicolumn{2}{|c|}{ No fire } & \multicolumn{2}{|c|}{ Fire } & \multicolumn{2}{|c|}{ No fire } & \multicolumn{2}{|c|}{ Fire } \\
\hline & & No tillage & Tillage & No tillage & Tillage & No tillage & Tillage & No tillage & Tillage \\
\hline \multicolumn{10}{|l|}{ Decreaser } \\
\hline Digitaria eriantha & $0.17 \pm 0.03$ & $0.16 \pm 0.03$ & $0.13 \pm 0.02$ & $0.53 \pm 0.04$ & $0.26 \pm 0.03$ & $0.20 \pm 0.04$ & $0.15 \pm 0.02$ & $0.80 \pm 0.09$ & $0.69 \pm 0.06$ \\
\hline $\begin{array}{l}\text { Helictotrichon } \\
\text { turgudulum }\end{array}$ & - & $0.32 \pm 0.02$ & - & $0.27 \pm 0.02$ & $0.17 \pm 0.03$ & - & $0.40 \pm 0.03$ & $0.40 \pm 0.03$ & - \\
\hline Panicum stapfianum & - & - & - & $0.27 \pm 0.03$ & - & - & - & $0.40 \pm 0.02$ & - \\
\hline $\begin{array}{l}\text { Pentaschistis } \\
\text { setifolium }\end{array}$ & - & - & - & $1.06 \pm 0.88$ & - & $0.78 \pm 0.68$ & - & - & - \\
\hline $\begin{array}{l}\text { Sporobolus } \\
\text { fimbriatus }\end{array}$ & $0.52 \pm 0.06$ & $0.64 \pm 0.05$ & $0.50 \pm 0.04$ & $0.66 \pm 0.03$ & $0.35 \pm 0.02$ & $0.78 \pm 0.03$ & $0.73 \pm 0.04$ & $0.80 \pm 0.05$ & $0.69 \pm 0.06$ \\
\hline Themeda triandra & $81.06 \pm 10.1$ & $2.56 \pm 0.9$ & $1.51 \pm 0.8$ & $1.32 \pm 0.7$ & $0.78 \pm 0.5$ & $2.54 \pm 0.9$ & $1.46 \pm 0.7$ & $1.60 \pm 0.6$ & $1.04 \pm 0.4$ \\
\hline \multicolumn{9}{|c|}{ 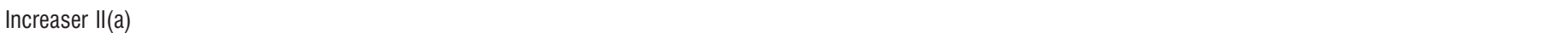 } & $2.42^{\mathrm{aba}} \pm 0.12$ \\
\hline $\begin{array}{c}\text { Cymbopogon } \\
\text { pospischilii }\end{array}$ & $1.21 \pm 0.9$ & $0.16 \pm 0.08$ & $0.13 \pm 0.06$ & $0.27 \pm 0.07$ & $0.17 \pm 0.03$ & $0.21 \pm 0.03$ & $0.15 \pm 0.04$ & $0.20 \pm 0.08$ & $0.17 \pm 0.08$ \\
\hline $\begin{array}{l}\text { Digitaria } \\
\quad \text { argyrograpta }\end{array}$ & $4.24 \pm 1.2$ & - & - & - & - & - & - & - & - \\
\hline $\begin{array}{l}\text { Eragrostis } \\
\quad \text { chloromelas }\end{array}$ & $8.13 \pm 1.9$ & $44.00 \pm 6.8$ & $27.64 \pm 7.3$ & $66.67 \pm 8.2$ & $36.14 \pm 7.1$ & $55.58 \pm 6.9$ & $29.87 \pm 3.4$ & $74.40 \pm 7.7$ & $42.63 \pm 5.6$ \\
\hline E. Iehmanniana & - & $0.64 \pm 0.09$ & $0.50 \pm 0.06$ & $0.93 \pm 0.04$ & $0.70 \pm 0.05$ & $0.39 \pm 0.03$ & $0.29 \pm 0.02$ & $0.20 \pm 0.02$ & $0.35 \pm 0.03$ \\
\hline E. superba & - & - & $0.50 \pm 0.04$ & $0.27 \pm 0.03$ & $1.04 \pm 0.06$ & $0.39 \pm 0.04$ & $4.39 \pm 0.9$ & $0.40 \pm 0.02$ & $2.08 \pm 0.9$ \\
\hline E. plana & - & - & $0.25 \pm 0.06$ & $0.27 \pm 0.06$ & - & - & - & - & - \\
\hline $\begin{array}{l}\text { Heteropogon } \\
\text { contortus }\end{array}$ & $0.28 \pm 0.06$ & - & - & $0.27 \pm 0.04$ & $0.17 \pm 0.02$ & - & - & - & - \\
\hline $\begin{array}{c}\text { Setaria spacelata } \\
\text { var. spacelata }\end{array}$ & $0.09 \pm 0.01$ & - & - & - & - & - & - & - & - \\
\hline \multicolumn{10}{|l|}{ Increaser II(b) } \\
\hline Agrostis lachmantha & - & - & $0.50 \pm 0.06$ & - & - & $0.39 \pm 0.06$ & - & - & - \\
\hline Bromus catharticus & - & - & - & - & - & - & - & - & - \\
\hline Cynodon hirsitus & - & - & - & - & - & $0.39 \pm 0.02$ & - & - & - \\
\hline Cynodon dactylon & - & - & - & - & - & $0.39 \pm 0.02$ & - & - & - \\
\hline Eragrostis obtusa & $0.09 \pm 0.01$ & $0.32 \pm 0.05$ & $0.25 \pm 0.03$ & $0.27 \pm 0.03$ & - & $0.39 \pm 0.04$ & $0.29 \pm 0.02$ & - & - \\
\hline Elionurus muticus & - & - & - & $1.57 \pm 0.06$ & - & - & - & - & - \\
\hline $\begin{array}{l}\text { Triraphis } \\
\quad \text { andropogonoides }\end{array}$ & $0.09 \pm 0.01$ & $0.64 \pm 0.07$ & - & - & - & - & - & - & - \\
\hline \multicolumn{10}{|l|}{ Increaser II(c) } \\
\hline Aristida congesta & $0.26 \pm 0.09$ & - & - & - & - & - & - & - & - \\
\hline Eleusinc coraeana & - & - & - & - & - & - & - & - & - \\
\hline $\begin{array}{l}\text { Eragrostis } \\
\quad \text { gummiflua }\end{array}$ & - & - & - & - & $0.27 \pm 0.09$ & - & - & $0.40 \pm 0.02$ & $0.36 \pm 0.05$ \\
\hline Lycium tenue & $1.12 \pm 0.09$ & - & - & - & - & - & - & - & - \\
\hline Tragus koelerioides & $1.47 \pm 0.99$ & - & - & - & - & - & - & - & - \\
\hline Walafrida saxatilis & $1.30 \pm 0.08$ & - & - & - & - & - & - & - & - \\
\hline \multirow[t]{2}{*}{ Increaser II total } & 18.25 & $45.76^{\mathrm{bab}}$ & $29.77^{\mathrm{bbb}}$ & $70.53^{\text {aаa }}$ & $38.58^{\mathrm{bba}}$ & $58.13^{\mathrm{aab}}$ & $34.99^{\mathrm{abb}}$ & $75.60^{\text {аaа }}$ & $45.59^{\mathrm{aba}}$ \\
\hline & \pm 0.21 & \pm 0.66 & \pm 0.33 & \pm 0.73 & \pm 0.23 & \pm 0.33 & \pm 0.22 & \pm 0.78 & \pm 0.52 \\
\hline \multirow[t]{2}{*}{ Weeds } & - & $50.56^{\mathrm{aba}}$ & $68.09^{\text {aаa }}$ & $25.37^{\text {aab }}$ & $59.95^{\text {aab }}$ & $37.57^{\mathrm{bba}}$ & $62.27^{\text {aаa }}$ & $20.40^{\mathrm{abb}}$ & $51.99^{\text {aab }}$ \\
\hline & & \pm 0.59 & \pm 1.12 & \pm 0.57 & \pm 0.66 & \pm 0.42 & \pm 1.11 & \pm 0.36 & \pm 1.23 \\
\hline
\end{tabular}


Table 1. Extended.

\begin{tabular}{|c|c|c|c|c|c|c|c|c|}
\hline \multirow{4}{*}{$\begin{array}{c}\text { Species (ecological } \\
\text { status) }\end{array}$} & \multicolumn{8}{|c|}{ Seedbank (2005/2006 growing season) } \\
\hline & \multicolumn{4}{|c|}{ Seed rain } & \multicolumn{4}{|c|}{ Blocked seed rain } \\
\hline & \multicolumn{2}{|c|}{ No fire } & \multicolumn{2}{|c|}{ Fire } & \multicolumn{2}{|c|}{ No fire } & \multicolumn{2}{|c|}{ Fire } \\
\hline & No tillage & Tillage & No tillage & Tillage & No tillage & Tillage & No tillage & Tillage \\
\hline \multicolumn{9}{|l|}{ Decreaser } \\
\hline Digitaria eriantha & $0.53 \pm 0.03$ & $0.45 \pm 0.03$ & $1.24 \pm 0.09$ & $1.70 \pm 0.09$ & - & - & - & - \\
\hline $\begin{array}{l}\text { Helictotrichon } \\
\text { turgudulum }\end{array}$ & $2.13 \pm 0.09$ & $1.35 \pm 0.08$ & $0.62 \pm 0.06$ & $0.57 \pm 0.04$ & - & - & - & - \\
\hline Panicum stapfianum & $0.53 \pm 0.06$ & - & - & - & - & - & - & - \\
\hline $\begin{array}{l}\text { Pentaschistis } \\
\text { setifolium }\end{array}$ & - & - & - & - & - & - & - & - \\
\hline $\begin{array}{l}\text { Sporobolus } \\
\text { fimbriatus }\end{array}$ & $1.07 \pm 0.08$ & $1.80 \pm 0.08$ & $0.62 \pm 0.04$ & $0.57 \pm 0.03$ & - & - & - & - \\
\hline Themeda triandra & $5.87 \pm 1.2$ & $5.39 \pm 1.2$ & $3.73 \pm 1.2$ & $3.40 \pm 0.9$ & - & - & - & - \\
\hline $\begin{array}{l}\text { Decreaser total } \\
\text { Increaser II(a) }\end{array}$ & $10.13^{-\mathrm{aa}} \pm 0.09$ & $8.99^{-b a} \pm 0.04$ & $6.21^{-a b} \pm 0.03$ & $6.24^{-a b} \pm 0.03$ & - & - & - & - \\
\hline $\begin{array}{l}\text { Cymbopogon } \\
\text { pospischilii }\end{array}$ & $0.27 \pm 0.02$ & $0.22 \pm 0.03$ & $0.62 \pm 0.06$ & $0.57 \pm 0.04$ & - & - & - & - \\
\hline $\begin{array}{l}\text { Digitaria } \\
\quad \text { argyrograpta }\end{array}$ & - & - & - & - & - & - & - & - \\
\hline $\begin{array}{l}\text { Eragrostis } \\
\quad \text { chloromelas }\end{array}$ & $25.07 \pm 3.9$ & $13.48 \pm 1.3$ & $22.36 \pm 4.2$ & $10.40 \pm 1.1$ & $4.83 \pm 2.6$ & $10.21 \pm 2.2$ & $33.83 \pm 3.7$ & $20.74 \pm 2.9$ \\
\hline E. lehmanniana & $1.60 \pm 0.8$ & $1.35 \pm 0.6$ & $4.97 \pm 0.9$ & $1.70 \pm 0.9$ & - & $0.73 \pm 0.08$ & - & - \\
\hline E. superba & $0.45 \pm 0.09$ & - & - & $1.34 \pm 0.9$ & - & $3.65 \pm 0.9$ & - & $2.22 \pm 0.9$ \\
\hline E. plana & - & $1.35 \pm 0.7$ & $0.62 \pm 0.03$ & - & - & - & - & - \\
\hline $\begin{array}{l}\text { Heteropogon } \\
\text { contortus }\end{array}$ & - & $0.90 \pm 0.04$ & - & $0.57 \pm 0.03$ & - & - & - & - \\
\hline $\begin{array}{c}\text { Setaria spacelata } \\
\text { var. spacelata }\end{array}$ & - & - & - & - & - & - & - & - \\
\hline \multicolumn{9}{|l|}{ Increaser II(b) } \\
\hline Agrostis lachmantha & - & - & - & - & - & - & - & $0.74 \pm 0.06$ \\
\hline Bromus catharticus & - & - & - & - & - & - & - & $0.74 \pm 0.06$ \\
\hline Cynodon hirsitus & - & - & - & - & - & - & - & - \\
\hline Cynodon dactylon & - & - & - & - & - & - & - & - \\
\hline Eragrostis obtusa & - & - & - & - & - & - & - & - \\
\hline Elionurus muticus & - & - & - & - & $0.69 \pm 0.06$ & $0.73 \pm 0.06$ & - & - \\
\hline $\begin{array}{l}\text { Triraphis } \\
\quad \text { andropogonoides }\end{array}$ & $2.13 \pm 0.09$ & $0.45 \pm 0.07$ & - & $1.34 \pm 0.09$ & - & $1.46 \pm 0.09$ & - & - \\
\hline \multicolumn{9}{|l|}{ Increaser II(c) } \\
\hline Aristida congesta & - & - & - & - & - & $0.73 \pm 0.06$ & - & - \\
\hline Eleusinc coraeana & - & - & - & - & - & - & $0.76 \pm 0.07$ & - \\
\hline $\begin{array}{l}\text { Eragrostis } \\
\quad \text { gummiflua }\end{array}$ & - & - & - & $0.57 \pm 0.06$ & - & - & - & $0.74 \pm 0.06$ \\
\hline Lycium tenue & - & - & - & - & - & - & - & - \\
\hline Tragus koelerioides & - & - & - & - & - & - & $0.76 \pm 0.09$ & $0.74 \pm 0.09$ \\
\hline Walafrida saxatilis & - & - & - & - & - & - & - & - \\
\hline Increaser II total & $\begin{array}{r}29.52^{\text {aаa }} \\
\pm 0.32\end{array}$ & $\begin{array}{r}17.75^{\mathrm{aba}} \\
\pm 0.12\end{array}$ & $\begin{array}{r}28.57^{\text {baa }} \\
\pm 0.32\end{array}$ & $\begin{array}{r}16.49^{\mathrm{bba}} \\
\pm 0.13\end{array}$ & $\begin{array}{r}25.52^{\mathrm{aab}} \\
\pm 0.42\end{array}$ & $\begin{array}{r}17.51^{\mathrm{abb}} \\
\pm 0.23\end{array}$ & $\begin{array}{r}34.59^{\text {aаa }} \\
\pm 0.52\end{array}$ & $\begin{array}{r}25.18^{\mathrm{aba}} \\
\pm 0.23\end{array}$ \\
\hline Weeds & $\begin{array}{r}60.35^{\mathrm{aba}} \\
\pm 1.44\end{array}$ & $\begin{array}{r}73.26^{\text {baa }} \\
\pm 1.52\end{array}$ & $\begin{array}{r}65.22^{\mathrm{aba}} \\
\pm 1.01\end{array}$ & $\begin{array}{r}77.27^{\text {aaa }} \\
\pm 0.99\end{array}$ & $\begin{array}{r}74.48^{\mathrm{aba}} \\
\pm 1.62\end{array}$ & $\begin{array}{r}82.49^{\text {aaa }} \\
\pm 1.99\end{array}$ & $\begin{array}{r}64.65^{\mathrm{abb}} \\
\pm 0.96\end{array}$ & $\begin{array}{r}74.08^{\mathrm{aab}} \\
\pm 1.01\end{array}$ \\
\hline
\end{tabular}


Table 2. Relative species frequency (\%) for the survived seedlings emerging from the soil samples collected from the rangeland for different treatments, which were germinating in the greenhouse. Seed bank soil collected in autumn for the 2002/2003 to 2006/2007 growing seasons. No Decreaser species occurred for blocked seed rain for the 2005/2006 and 2006/2007 seasons and therefore the gaps. Within a treatment (row) and ecological status, different letters indicate significant $(P<0.05)$ differences, between years based on the Tukey test.

\begin{tabular}{|c|c|c|c|c|c|c|c|c|}
\hline \multirow[b]{3}{*}{ Seasons and ecological status } & \multicolumn{4}{|c|}{ Seed rain } & \multicolumn{4}{|c|}{ Blocked seed rain } \\
\hline & \multicolumn{2}{|c|}{ No fire } & \multicolumn{2}{|c|}{ Fire } & \multicolumn{2}{|c|}{ No fire } & \multicolumn{2}{|c|}{ Fire } \\
\hline & No tillage & Tillage & No tillage & Tillage & No tillage & Tillage & No tillage & Tillage \\
\hline \multicolumn{9}{|l|}{$2002 / 2003$} \\
\hline Decreaser & $3.68^{c}$ & $2.14^{b}$ & $4.11^{\mathrm{b}}$ & $1.56^{c}$ & $4.30^{c}$ & $2.74^{b}$ & $4.00^{\mathrm{a}}$ & $2.42^{b}$ \\
\hline Increaser II a & $44.80^{a}$ & $29.02^{b}$ & $68.68^{\mathrm{a}}$ & $38.22^{b}$ & $56.57^{\mathrm{a}}$ & $34.70^{a}$ & $75.20^{a}$ & $45.23^{b}$ \\
\hline Increaser II b & $0.96^{b}$ & $0.75^{b}$ & $1.84^{\mathrm{a}}$ & 0 & $1.56^{\mathrm{a}}$ & $0.29^{b}$ & 0 & 0 \\
\hline Increaser II c & 0 & 0 & 0 & $0.27^{c}$ & 0 & 0 & $0.40^{b}$ & $0.36^{a}$ \\
\hline Weeds & $50.56^{a}$ & $68.09^{a}$ & $25.37^{\mathrm{b}}$ & $59.95^{b}$ & $37.57^{c}$ & $62.27^{c}$ & $20.40^{b}$ & $51.99^{b}$ \\
\hline \multicolumn{9}{|l|}{$2003 / 2004$} \\
\hline Decreaser & $6.72^{b}$ & $8.72^{\mathrm{a}}$ & $3.64^{b}$ & $4.62^{b}$ & $6.86^{a}$ & $5.10^{\mathrm{a}}$ & $3.47^{\mathrm{a}}$ & $4.24^{\mathrm{a}}$ \\
\hline Increaser II a & $57.84^{c}$ & $47.70^{a}$ & $78.02^{\mathrm{a}}$ & $72.15^{a}$ & $57.23^{a}$ & $34.71^{\mathrm{a}}$ & $76.08^{a}$ & $62.05^{\mathrm{a}}$ \\
\hline Increaser II b & $0.81^{\mathrm{b}}$ & $1.45^{\mathrm{a}}$ & $1.26^{a}$ & 0 & $1.26^{a}$ & $0.11^{\mathrm{b}}$ & 0 & 0 \\
\hline Increaser II c & 0 & 0 & 0 & $0.29^{c}$ & 0 & $0.48^{a}$ & $0.40^{\mathrm{b}}$ & $0.29^{c}$ \\
\hline Weeds & $34.63^{b}$ & $42.13^{b}$ & $17.08^{b}$ & $22.94^{c}$ & $34.65^{c}$ & $59.60^{c}$ & $20.05^{b}$ & $33.42^{\mathrm{C}}$ \\
\hline \multicolumn{9}{|l|}{ 2004/2005 } \\
\hline Decreaser & $7.58^{\mathrm{b}}$ & $6.56^{\mathrm{a}}$ & $4.23^{b}$ & $4.11^{\mathrm{b}}$ & $5.67^{\mathrm{a}}$ & $6.57^{\mathrm{a}}$ & $4.25^{\mathrm{a}}$ & $4.71^{\mathrm{a}}$ \\
\hline Increaser II a & $28.78^{b}$ & $13.11^{c}$ & $31.09^{b}$ & $18.12^{c}$ & $27.67^{b}$ & $19.14^{b}$ & $31.68^{b}$ & $17.78^{c}$ \\
\hline Increaser II b & $0.04^{c}$ & $0.42^{c}$ & $0.50^{b}$ & $0.48^{\mathrm{b}}$ & $1.33^{\mathrm{a}}$ & $0.11^{\mathrm{b}}$ & 0 & $0.47^{\mathrm{c}}$ \\
\hline Increaser II C & 0 & 0 & 0 & $1.44^{\mathrm{a}}$ & 0 & $0.58^{\mathrm{a}}$ & $0.40^{b}$ & $0.95^{b}$ \\
\hline Weeds & $63.60^{a}$ & $79.91^{\mathrm{a}}$ & $64.18^{a}$ & $75.85^{a}$ & $65.33^{b}$ & $73.6^{b}$ & $63.67^{\mathrm{a}}$ & $76.09^{a}$ \\
\hline \multicolumn{9}{|l|}{ 2005/2006 } \\
\hline Decreaser & $10.13^{\mathrm{a}}$ & $8.99^{a}$ & $6.21^{a}$ & $6.24^{\mathrm{a}}$ & 0 & 0 & 0 & 0 \\
\hline Increaser II a & $27.39^{b}$ & $17.30^{c}$ & $28.57^{b}$ & $14.58^{c}$ & $4.83^{c}$ & $14.59^{b}$ & $33.83^{b}$ & $23.96^{\mathrm{c}}$ \\
\hline Increaser II b & $2.13^{\mathrm{a}}$ & $0.45^{c}$ & 0 & $1.34^{\mathrm{a}}$ & $0.69^{b}$ & $2.19^{a}$ & 0 & $1.48^{b}$ \\
\hline Increaser II c & 0 & 0 & 0 & $0.57^{\mathrm{b}}$ & 0 & $0.73^{\mathrm{a}}$ & $1.52^{\mathrm{a}}$ & $1.48^{a}$ \\
\hline Weeds & $60.35^{\mathrm{a}}$ & $73.26^{a}$ & $65.22^{a}$ & $77.27^{\mathrm{a}}$ & $74.48^{b}$ & $82.49^{a}$ & $64.65^{\mathrm{a}}$ & $74.08^{a}$ \\
\hline \multicolumn{9}{|l|}{ 2006/2007 } \\
\hline Decreaser & $12.64^{\mathrm{a}}$ & $7.33^{a}$ & $7.80^{a}$ & $8.00^{a}$ & 0 & 0 & 0 & 0 \\
\hline Increaser II a & $35.04^{b}$ & $15.09^{c}$ & $29.88^{b}$ & $10.40^{c}$ & $8.23^{c}$ & $13.22^{b}$ & $29.17^{b}$ & $17.49^{c}$ \\
\hline Increaser II b & $1.28^{a}$ & $0.11^{d}$ & 0 & $1.60^{\mathrm{a}}$ & $1.18^{a}$ & $0.88^{b}$ & 0 & $4.60^{\mathrm{a}}$ \\
\hline Increaser II c & 0 & 0 & 0 & $0.05^{b}$ & 0 & $0.72^{\mathrm{a}}$ & $2.08^{a}$ & $0.57^{b}$ \\
\hline Weeds & $51.04^{\mathrm{a}}$ & $77.47^{\mathrm{a}}$ & $62.32^{\mathrm{a}}$ & $79.50^{a}$ & $90.59^{a}$ & $85.18^{a}$ & $68.15^{\mathrm{a}}$ & $77.34^{\mathrm{a}}$ \\
\hline
\end{tabular}

\section{RESULTS}

\section{Botanical Composition of Vegetation in the Field}

The rangeland was dominated by Themeda triandra before applying the treatments (2002/2003 growing season), which constituted $81 \%$ of the field's species composition (Table 1 ). This botanical composition clearly showed that the field was in a good condition at the onset of the study (Snyman 2000). Interestingly, no weeds occurred in the field. Floristic composition did not vary for any treatment over the five growing seasons (data not shown).

\section{Seed Bank Germination in the Greenhouse Following Disturbance}

Disturbance is defined as the physical impact on the plant and soil through fire, trampling, and seed removal. Botanical compositions of the surviving seedlings for every treatment in the soil seed bank for only the autumn germination are presented in Table 1. Adding different season's seed bank data is not convenient, since the same seed may be counted several times (at least for a persistent seed bank). Only the species composition of the seed bank, as obtained for the 2002/2003 growing season and that after four growing seasons applying the treatments (2005/2006), are presented in Table 1. The reason for this being that after four growing seasons, no Decreaser grass species germinated in the seed bank with seed removal (Table 2). In contrast, the frequency of Increaser species and weeds showed an enormous variation between the five growing seasons (Table 2).

\section{Seed Bank Composition and Species Richness}

After only four growing seasons of applying the blocked seed rain (removing seeds) treatment, no further seedling emergence of the Decreaser species occurred in the seed bank (Table 2). 
This cessation in seedling emergence was a marked $(P<0.05)$ occurrence as the previous growing season (2004/2005) was characterized by seedling emergence in the seed bank in all the Decreaser species regardless of the treatment (Table 2). This tendency only occurred among the Decreaser species, while most Increaser species showed almost the same $(P>0.05)$ germination after four seasons of blocked seed rain, compared with that of the seed rain treatment, regardless of the other treatments applied (Table 1). Among the Increaser species, Cymbopogon pospischilii, Eragrostis plana, and Heteropogon contortus in particular had almost no seedling emergence after four seasons of blocked seed rain. Notably, the germination of E. chloromelas, regardless of the other two treatments, was not at all influenced by blocked seed rain after $4 \mathrm{yr}$ and this still had a high seed presence in the seed bank (Table 1).

Emergence of both Decreaser and Increaser species seedlings were very sensitive to the tillage treatment $(P<0.05)$, and regardless of the other treatments, gave rise to a significant decrease in seedlings in the seed bank $(P \leq 0.05)$. By contrast, the weeds increased significantly in seedling emergence with the application of the tillage treatment $(P<0.05)$, regardless of the other two treatments. This was the tendency for most growing seasons (Table 2).

The fire treatment, applied once (2002/2003 growing season) 4 yr later, still caused a decrease $(P<0.05)$ in seedling emergence in the seed bank of the Decreaser species, regardless of the other treatments applied (Tables 1 and 2). The germination of the Increaser species was generally stimulated by the fire treatment $(P<0.05)$. Themeda triandra was the most sensitive towards burning (Table 1).

No significant $(P>0.05)$ interaction was obtained for all growing seasons for species frequency among the combined three factors (seed rain, tillage, and fire), for Decreaser, Increaser, and weed species (Tables 1 and 2). A significant interaction was obtained for all growing season for species frequency among tillage and fire for the Decreaser and weed species $(P<0.05$; Tables 1 and 2$)$.

The species Digitaria argyrograpta, Setaria sphacelata var. sphacelata, Lycium tenue, and Walafrida saxatilis (4/27 species) only occurred in the vegetation and never in the soil seed bank over the five growing seasons. Those species only occurring in the seed bank over the $5 \mathrm{yr}$, but not in the vegetation, included Helictotrichon turgidulum, Panicum stapfianum, Pentaschistis setifolium, E. lehmanniana, E. superba, E. plana, Agrostis lachnantha, E. gummiflua, Cynodon dactylon, C. hirsutus, Elionurus muticus, Bromus catharticus, and Eleusine coracana (13/27 species). The weed species were not accounted for in the above species richness. The study years not shown in Table 1 had no additional species in the seed bank or did not occur in the vegetation or vice versa.

There was no significant $(P>0.05)$ interaction in terms of species richness between the combined tillage, blocked seed rain, and fire treatments (data not shown). On the other hand, species richness significantly $(P<0.05)$ declined over time for the seed rain treatment over the last $4 \mathrm{yr}$ of applying it, regardless of the other treatments (Fig. 1). Notably, for the blocked seed rain treatment over the five studied years, the species richness was significantly $(P<0.05)$ lower for all the years than in seed rain treatments.

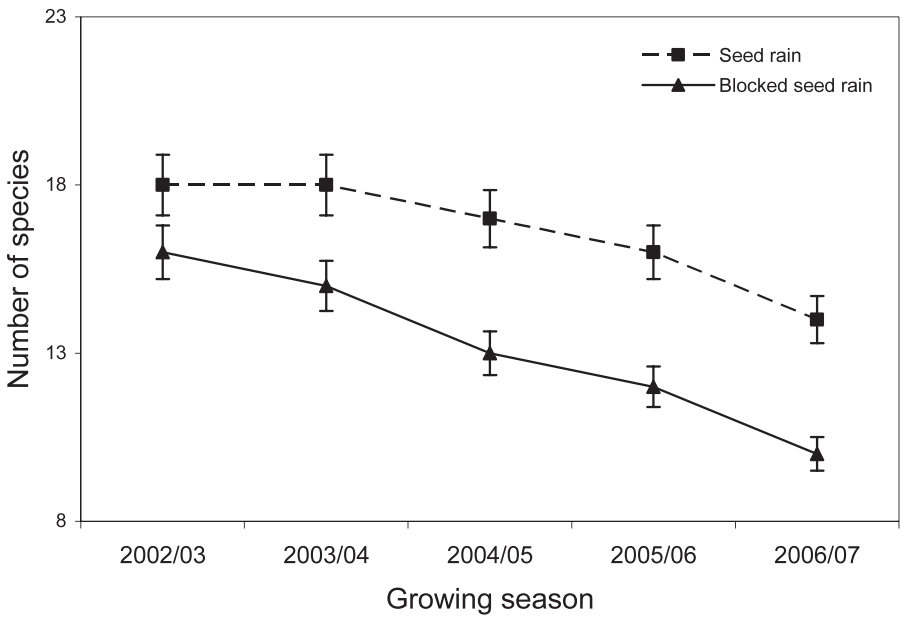

Figure 1. Species richness (mean number of grass species) for the blocked seed rain treatment regardless of tillage or fire for the 2002/2003 to $2006 / 2007$ growing seasons. Error bars are the standard error of means $(n=3)$, where they are significantly different $(P<0.05)$. $\mathrm{LSD}_{0.01}=1.3$.

\section{Vegetation Similarity Between Seed Bank and Abovegound (Field) Prior to Disturbance}

In the rangeland (field), relatively low $(P>0.05)$ similarity was detected between species composition in the vegetation and in the seed bank for most treatments (ranged from $53.7 \%$ to $38.1 \%$; except the control; 68.6\%), $4 \mathrm{yr}$ following the application of the different treatments. When the high $(P<0.05)$ value of the control (no seed removal, no burning, and no tillage) of $68.6 \%$ was compared with the other treatments (averaged $45.1 \%$ ), it was clear that all treatments impacted vegetation similarity. As expected, where seeds were removed (averaged 40.6\%) compared to seed rain (averaged $59.2 \%)$, the similarity indexes were the lowest $(P>0.05)$ regardless of the other treatments.

\section{Seed Bank Density}

As expected, the blocked seed rain treatment caused a significant decrease $(P<0.05)$ in seed bank density for all seasons (Table 3). Spring and summer tillage did not influence seed bank density significantly $(P<0.05)$, but significantly $(P<0.05)$ increased it for the autumn seed collection. Fire significantly $(P<0.05)$ increased the seed bank density for all seasons when tillage took place and seed rain was allowed. Over the first two seasons, there was a nonsignificant interaction for seed density among seed rain, tillage, and fire treatments $(P>0.05)$. A combined tillage, fire, and seed rain treatment showed the high seed bank density.

Fire significantly $(P<0.05)$ stimulated seed bank density over the first two growing seasons, regardless of the other treatments applied (Fig. 2A). As burning was a single occurrence, it could be expected to influence seed bank density nonsignificantly $(P>0.05)$ after the second growing season. The blocked seed rain treatment caused a decrease $(P<0.05)$ in seed bank density over all growing seasons as expected (Fig. 2B). Those plants that seeded (seed rain) showed a variable seed bank density over the $5 \mathrm{yr}$ with especially favorable seed bank density in the 2003/2004 growing season. Tillage increased 
Table 3. Mean seed bank density (mean number of live seedlings $\cdot \mathrm{m}^{-2}$ ) obtained by the seedling emergence method from soil samples of rangeland for different treatments. Soil samples were taken in spring (October), summer (January), and autumn (April) for the 2002/2003 to 2006/2007 growing seasons, and were germinated in the greenhouse. Data are means and standard errors. Within a season, different first letters indicate significant $(P<0.05)$ differences among blocked seed rain treatments within each fire treatment, different second letters indicate significant $(P<0.05)$ differences among tillage treatments within each fire treatment, and different third letters indicate significant $(P<0.05)$ differences between fire treatments within a blocked seed rain treatment, based on the Tukey test.

\begin{tabular}{|c|c|c|c|c|c|c|}
\hline & \multicolumn{2}{|c|}{ Spring } & \multicolumn{2}{|c|}{ Summer } & \multicolumn{2}{|c|}{ Autumn } \\
\hline & Seed rain & Blocked seed rain & Seed rain & Blocked seed rain & Seed rain & Blocked seed rain \\
\hline \multicolumn{7}{|l|}{ No fire } \\
\hline No tillage & $132.8^{\mathrm{aab}} \pm 10.16$ & $86.2^{\mathrm{bab}} \pm 4.14$ & $242.2^{\text {aaa }} \pm 39.12$ & $186.8^{\text {baa }} \pm 21.20$ & $128.8^{\mathrm{aba}} \pm 20.14$ & $79.4^{\mathrm{bba}} \pm 6.26$ \\
\hline Tillage & $140.0^{\mathrm{aab}} \pm 11.14$ & $107.4^{\text {baa }} \pm 9.15$ & $244.4^{\mathrm{aab}} \pm 41.21$ & $187.2^{\text {baa }} \pm 26.15$ & $162.8^{\mathrm{aab}} \pm 20.14$ & $137.2^{\text {baa }} \pm 21.14$ \\
\hline \multicolumn{7}{|l|}{ Fire } \\
\hline No tillage & $157.0^{\text {aaa }} \pm 21.21$ & $97.0^{\mathrm{baa}} \pm 9.14$ & $268.8^{\text {aaa }} \pm 42.16$ & $168.6^{\text {baa }} \pm 31.11$ & $137.2^{\mathrm{aba}} \pm 28.14$ & $85.6^{\mathrm{bba}} \pm 9.12$ \\
\hline Tillage & $157.2^{\text {aаa }} \pm 31.21$ & $98.0^{\text {baa }} \pm 6.66$ & $294.4^{\text {aaa }} \pm 42.14$ & $186.0^{\text {baa }} \pm 21.41$ & $197.6^{\text {aаa }} \pm 29.11$ & $126.8^{\text {baa }} \pm 23.16$ \\
\hline
\end{tabular}
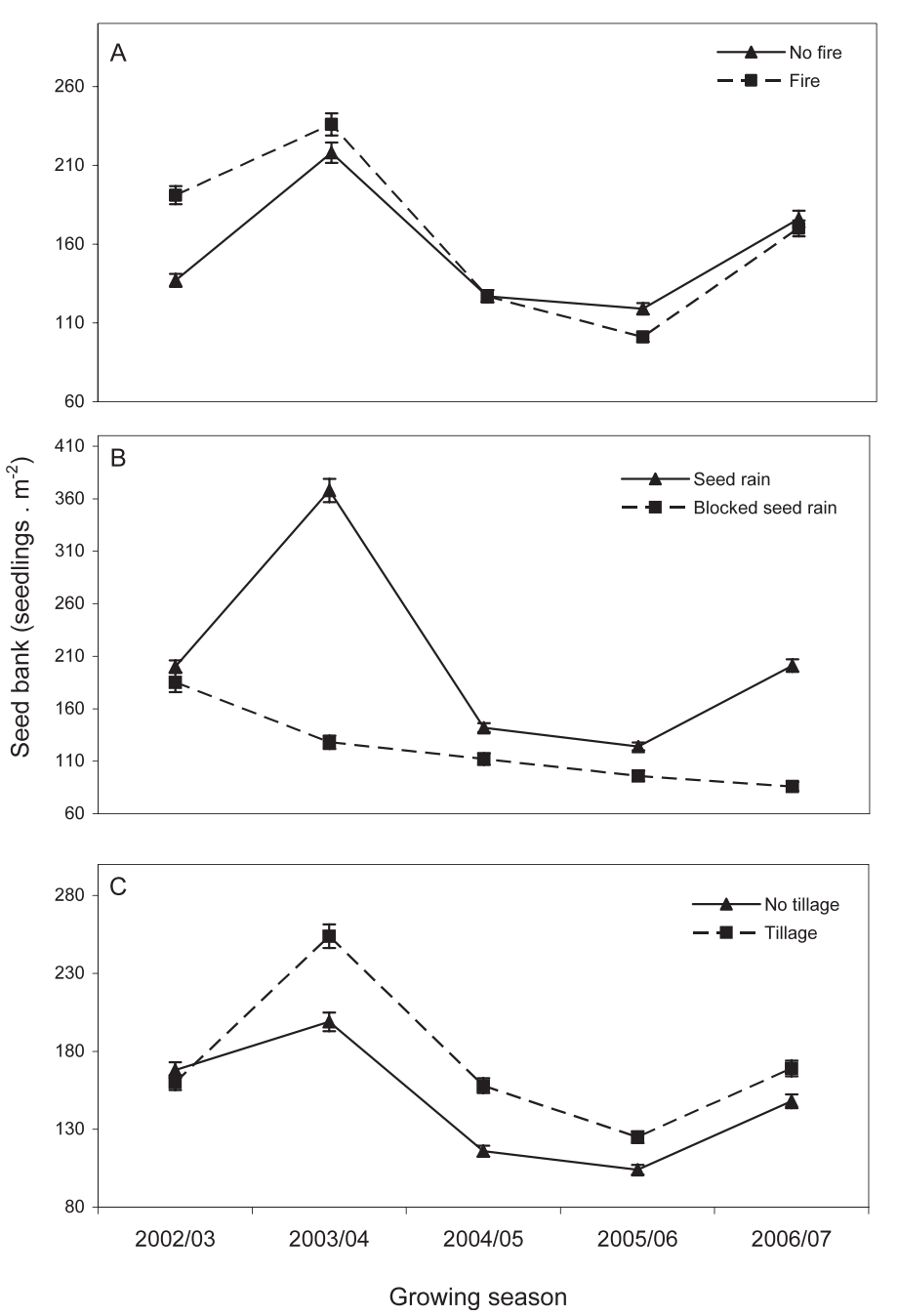

Figure 2. Mean seed bank density (number of live grass seedlings $\cdot \mathrm{m}^{-2}$ ) for the fire (regardless of blocked seed rain or tillage; $\mathbf{A}$, blocked seed rain (regardless of tillage or fire; $\mathbf{B}$, and tillage (regardless of fire or blocked seed rain; C, treatments for the 2002/2003 to 2006/2007 growing seasons. Vertical bars are standard errors of means $(n=3)$, where they are significantly different $(P<0.05) . \quad \mathrm{LSD}_{0.01}$ : fire $=10.2$; blocked seed rain $=10.3$; and tillage $=14.6$. seed bank density from the second to the fifth year, regardless of the other treatments (Fig. 2C).

\section{Regeneration of Vegetation Following Disturbance (2002/2003 Growing Season)}

The year 2002/2003, when the rainfall was not only well distributed but almost similar to the long-term average for the area, showed a high seedling emergence for different grass species in the field. This growing season, characterized by three periods of germination over the year (spring, summer, and autumn), is fully discussed for all the treatments (Table 4). Regardless of the treatments applied and species, a high germination occurred during spring, a few months following disturbance, on average $119 \%$ more than the other two seasons. A very small variety of grass species germinated in the field with surprisingly no annual weeds or forbs. Tillage increased total seedling emergence significantly $(P<0.05)$, regardless of the other two treatments applied (Table 4). Tillage significantly increased $(P<0.05)$ perennial grasses seedling emergence, especially Themeda triandra. It was evident that fire stimulated total seedling emergence for all seasons, for all the different grass species.

Although the blocked seed rain treatment decreased total seedling emergence significantly as expected over the 2002/ 2003 growing season $(P<0.05)$, regardless of the other treatments (Table 4), its effect was not as drastic as it was in the fourth year of applying the treatment. The exception was the increase $(P<0.05)$ in seedling emergence for the blocked seed rain treatment during spring for Eragrostis chloromelas. As the rainfall was not only low, but also lacked a high distribution pattern over the last 4 yr (2003/2004 to 2006/ 2007), seedling emergence was variable within a year.

A significant $(P>0.05)$ interaction was obtained for seedling density among the combined three factors (seed rain, fire, and tillage). There was a significant interaction $(P<0.05)$ obtained for seedling density among tillage and fire, only for spring of the 2002/2003 growing season. On the other hand, both summer and autumn seedling density show a significant interaction $(P<0.05)$ among seed rain and fire treatments. 


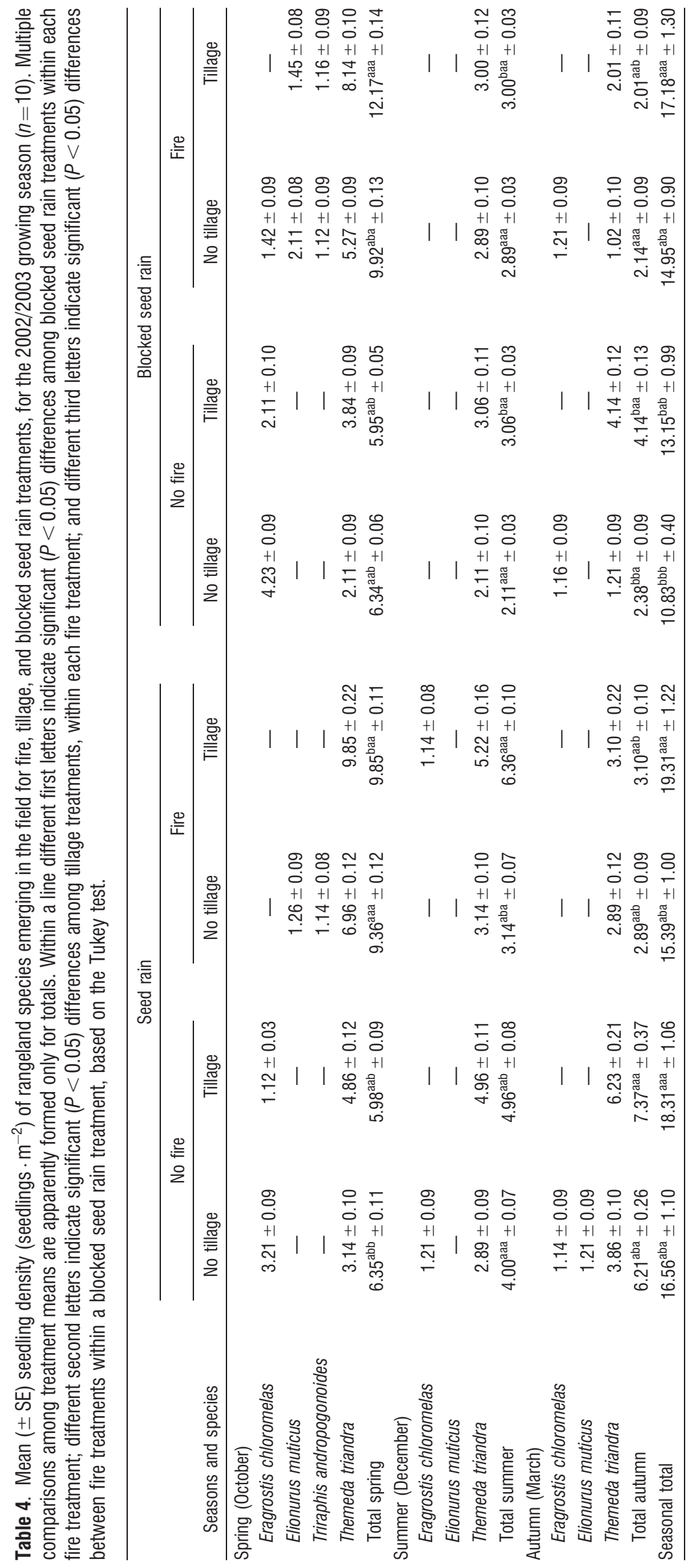




\section{DISCUSSION}

\section{Longevity of Seeds in Seed Bank}

This study clearly showed that the longevity of the Decreaser grass species seeds were very poor as after only $4 \mathrm{yr}$ of seed removal (blocked seed rain, as simulation of intensive grazing), no more seedlings emerged from the seed bank. It was not possible that the soil samples could have missed some viable seeds. The blocked seed rain experiment therefore support the posed hypotheses and contention that Themeda triandra (O'Connor 1997) and the other Decreaser species are prone to failed seedling recruitment under sustained defoliation, because of elimination of the seed bank (Auffret and Cousins 2011). In contrast, Themeda triandra showed considerable annual seed turnover under seed rain conditions (Table 1). If Decreaser grass species cannot seed for whatever reason in this semi-arid rangeland, the potential of the seed bank decreases linearly until it is suddenly depleted after 4 yr. At that time, only 11 out of 27 grass species were present in the seed bank where seeds were permanently removed. The remaining grasses in the seed bank were those with very small seeds (Eragrostis species mainly). With the few Decreaser species (Helictrotrichon turgidulum and Panicum stapfianum) present in the seed bank and not in the vegetation, the plant community is at risk to cross a threshold into an alternative stable state from which it is unable to revert without active intervention. Further more, the seed bank is also dominated by many Increaser species not present in the vegetation, which can also contribute to a threshold for recovery. According to O'Connor (1997), T. triandra does not have a seed bank older than $1 \mathrm{yr}$, which is debatable when compared with these results. In a Montane grassland of South Africa, high predation of seeds (70-98\%) and low viability ( $37 \%$ in 15 -month-old seeds) contributed to poor representation of $T$. triandra in the seed bank $(<1.2 \%)$ when compared with the aboveground vegetation $(<29.2 \%$; Everson et al. 2009). These attributes, together with its poor dispersal, indicate that T. triandra will only become reestablished over long periods of time or with major management inputs (Everson et al. 2009). The availability of seed therefore depends on seed production by the established population in the preceding year (O'Connor and Pickett 1992) and also the maintenance of its viability in the seed bank over time (Wassie and Teketay 2006).

The Eragrostis species, especially Eragrostis chloromelas, still had a good seedling emergence in the seed bank after $4 \mathrm{yr}$ of removing the seeds from the plants (blocked seed rain). Sheer numbers of other Increaser species, such as E. lehmanniana, E. superba, E. plana, E. gumniflua, Cynodon dactylon, C. hirsutus, and Elionurus muticus only occurring in the seed bank but not in the vegetation, might overpower the few Decreasers and can later dominate the community. This would imply nonreversibility of a transitional state. Due to Eragrostis species production of numerous, long-lived seeds as part of their opportunistic strategy, they usually predominate in seed banks (du Toit and Alard 1995). The very small seeds of the Eragrostis species therefore have good survival ability. The dominance of Eragrostis species after $4 \mathrm{yr}$ in the seed bank is supported by studies finding that small seeds, like those of Eragrostis spp., dominate because they can bury into the soil faster and escape predation (Thompson et al. 1993; Funes et al.
1999). According to Jones (1968), Eragrostis species (Increaser species) can yield an immense quantity of seed (up to 21000 seeds $\left.\cdot \mathrm{m}^{-2}\right)$, which exceeded that of T. triandra by far (138 seeds $\cdot \mathrm{m}^{-2}$; Snyman 2004). Perennial grasses, especially the larger-seeded species, do not, in general, form persistent seed banks even in the absence of seed predation, because of poor seed survival (Fenner and Thompson 2005). Snyman (2010) also argued that the larger a grass seed the shorter its longevity. These results also supports the findings of Cabellero et al. (2008) in semi-arid Mediterranean rangelands, where the temporal relationship in abundance of perennials between seed bank and vegetation was weaker than for annuals, as the local persistence of perennials is mainly based on the plant's longevity. Olano et al. (2005) conclude that semi-arid seed banks are a good predictor of annual community performance in terms of vegetation composition, which is confirmed by my results. Therefore, in the seed bank, time influenced the survival of grasses with larger seeds the most.

\section{Disturbance and the Soil Seed Bank}

The soil seed bank was largely dominated by Increaser grass species, while seedlings established in the field were more dominated by Decreaser grasses. The disturbed habitat in the seed bank was therefore more beneficial for species with a lower ecological status. The same explosion of pioneer plants is found in practice when soil is cultivated or mechanically disturbed for the first time (Snyman 2003). By contrast, Bekker et al. (1997) found that intense cultivation reduces the heterogeneity of seed banks within the soil, thereby delaying recovery of floristically diverse rangelands. As hypothesized, Decreaser grass species prefer a more natural and undisturbed habitat for successful establishment and survival (Snyman 2003). According to Wolfson and Tainton (1999), the relative importance of seed to the survival of T. triandra populations varies according to the ecological conditions of the site. Seeds of perennial grasses and more specific Decreaser species are usually scarce in the soil and this would explain, at least partly, the slow recovery of disturbed perennial rangelands (Amaha Kassahun et al. 2009).

Not only did the Decreaser species frequency decrease drastically after $4 \mathrm{yr}$ of seed removal (blocked seed rain), but so did the species richness, as suggested in the hypotheses posted. The decrease in species richness with seed rain (Fig. 1) can be ascribed to the influence of the other two treatments (burning and tillage) on the seed bank. Both Decreaser and Increaser species were very sensitive to the tillage treatment (intended as a trampling surrogate), which is supported by negative trampling influences on species richness of seed banks reported for a Mediterranean coastal sand dune community (Yu et al. 2008). By contrast, O'Connor et al. (2010) and Rutherford and Powrie (2011) reported significant increases in plant species richness with heavy grazing under communal grazing systems.

Fire applied once-off reduced the Decreaser species in the seed bank. Although fire still caused a decrease in Decreaser seedling emergence in the seed bank after $4 \mathrm{yr}$, the survived Decreaser species in the vegetation will compete sufficiently with the Increaser species to return the community to predisturbance composition. This would not apply the crossing 
of a definite threshold. In particular, T. triandra was the most sensitive towards fire, as noted by Zacharias et al. (1989). This response in T. triandra is in direct contrast to the generally held view that this species evolved under a consistent fire regime (O'Connor 1997). On the other hand, T. triandra seedlings emerge most readily when the seeds lie on the soil surface (O'Connor 1997). However, it has also been suggested that because of the sensitivity of this species' seeds to fire, it could be postulated that reproduction of $T$. triandra is achieved primarily by vegetative reproduction (Zacharias et al. 1989). Burning significantly stimulated seedling density of $T$. triandra in the seed bank over the first two seasons following the fire, as supported by the findings of Snyman (2005) on the same rangeland type. A significant interaction for seed bank density among fire and seed rain was also obtained over the last three seasons after applying the different treatments.

The relatively low seed densities found in this perennial rangeland are similar to genuine rangeland, which usually displays smaller seed banks (Bakker 1989; O’Connor 1997; Snyman 2005; Amaha Kassahun et al. 2009; Everson et al. 2009) than that of arable lands (Jensen 1969). Caution is necessary when comparing studies of soil seed banks because of different methods (Dreber 2011); e.g., with respect to the sample size, depth, time, and number of germination cycles (Koch et al. 2011). In the perennial rangeland prior to disturbance, the seed bank was dominated by early successional species, while conversely the aboveground vegetation was mainly dominated by perennial grasses. Accepting that the seed banks were persistent, the question remains as to whether they reflect the composition of the existing standing herbage or, if they do not, what the cause might be. According to Page et al. (2006), one of the main reasons could be that much of the seed bank sampling in the past may have been inadequate. The general trend is that the seed bank played only a minor role in recruitment into gaps in the field in the semi-arid rangelands, which is similar to findings in a few other studies (Edwards and Crawley 1999; Snyman 2005; Jacquemyn et al. 2011; Koch et al. 2011). For perennial rangelands, soil seed banks act as an important reservoir and buffer, but restoration from seed banks alone is very unlikely in degraded rangelands (Koch et al. 2011).

As expected, the blocked seed rain treatment caused a significant decrease in seed bank density for all seasons. However, many researchers argued that overgrazing, trampling, or removal of vegetation by animals can have complex effects on the number of seeds produced by a plant and released as seed rain (Page and Beeton 2000), which can both increase (Savory and Pearson 1980; Froud-Williams et al. 1984; Adams 1996; Snyman 2004, 2005) or decrease (Kinloch and Friedel 2005; Solomon et al. 2006; Amaha Kassahun 2009) seed bank density. The large seed bank existing during rangeland degradation is unfortunately dominated in most cases by less palatable grass species, which are also not very strongly perennial and will therefore only temporarily stabilize the soil (Snyman 2004).

In the present study, 13 grass species were found that only occurred in the seed bank and not in the standing vegetation (Table 1). Most of these prefer a moist habitat in the field: Eleusine coracana, Agrostis lachnantha, Bromus catharticus, Eragrostis species, Panicum stapfianum, and Helictotrichon turgidulum or disturbed conditions, Cynodon dactylon, C. hirsutus, and Pentaschistis setifolium. This suggests that the potential for the vegetation composition to change through recruitment from a seed bank is high. An alternative way in which recruitment might contribute to vegetation change is if the recruited seedlings give rise to a different composition than that of the existing vegetation, as was the case with this study. Only a few species were present in the vegetation and absent in the soil seed bank: mostly shrub species. Other researchers also argued that restoration of unimproved or semi-natural rangeland cannot always depend on a high level of recruitment from the seed bank of species that have disappeared from the established vegetation (Bekker et al. 1997; de Villiers et al. 2003; Lemenih and Teketay 2006; Ma et al. 2010; Dreber 2011). By contrast, a few researchers have reported on the very high similarity between soil seed banks and aboveground vegetation in communities (Levassor et al. 1991; Peco et al. 1998). Disturbance like intense grazing can also increase the similarity in species composition between soil seed banks and aboveground vegetation (Luzuriage et al. 2007).

\section{Seedling Recruitment Into Gaps in the Field}

Although theoretical models predict that moderate grazing might enhance species diversity compared with ungrazed lands factors influencing biodiversity are still incompletely understood (Olff and Ritchie 1998). In studies where grazing was excluded from semi-arid rangelands, it was found that perennial grasses increased initially, resulting in a rapid decline in species diversity (Hill et al. 1992). The absence of a grazing effect might be a function of the proportion of persistent and transient seeds and time of sampling (Kinucan and Smeins 1992). By contrast, in the present study the tillage action led to a significant increase in perennial grasses, especially Themeda triandra (Table 4). If the tillage treatment applied in this study can be viewed as the "herd effect" of a holistic resource management approach (Savory 1999), it could be an important management tool (animal impact), because of its supposed beneficial effect on the soil (Froud-Williams et al. 1984). It is envisaged that hoof action will break up the top soil layer (Zacharias et al. 1998). However, current understanding indicates that the benefits of hoof action are very limited: only for a short period and only on certain soil types (du Toit et al. 2008) and that the deleterious effects of soil compaction are more pronounced and persist for long periods (Busby and Gifford 1987; Wilcox and Wood 1988). Other researchers also reported of both positive and negative seedling recruitment benefits within functional groups following rangeland trampling (Kinloch and Friedel 2005; Yu et al. 2008; O'Connor et al. 2010; Rutherford and Powrie 2011).

Themeda triandra was the grass species with the best seedling emergence in the gaps in the field regardless of the treatment applied. This is not surprising because T. triandra was the dominant species in the field, which constituted $81 \%$ of the field's species composition before applying the treatments. The study shows that a few surviving Decreaser species compete sufficiently with the Increasers and early seral species to return the community to predisturbance composition and will not be overpowered by sheer numbers of Increasers in the soil seed bank and fade from the community. The soil seed 
bank forms therefore a potential pool of propagules for the regeneration of aboveground vegetation after disturbance (Bekker et al. 1997; Dreber et al. 2011), and reduces the probability of population extinction in the aboveground vegetation (Venable and Brown 1988). Based on to the species composition in the field before applying the treatments, only four species' seedlings emerged in the gaps, which is supported by Snyman (2004) who argue that recruitment from the seed bank and seed set may be more important in vegetation change when large-scale gaps arise. Studies have also shown that plant cover can provide particular environmental conditions that meet species-specific requirements for germination, so that certain species recruit within gaps regardless of the species composition of the seed bank or the seed set (Rusch and Fernàndez-Palacios 1995).

It was also clear that burning of this semi-arid rangeland had an important impact on the survival of seedlings in the field. The first $2 \mathrm{yr}$ following the fire, seedling emergence in the field was stimulated by burning after which it drastically declined because of loss of available gaps. Also only at the beginning of the season a positive interaction was obtained for seedling density among fire, seed rain, and tillage treatment. Unfortunately, information on the influence of fire on the seed bank of grassland is lacking in the literature, because most studies have concentrated on savannah regions. However, fire intensity influences on seed survival and emergence were qualified for Argentine and South African rangelands (Everson 1999; Laterra et al. 2006; Snyman 2006).

\section{IMPLICATIONS}

The study outlines the importance of considering several characteristics of seed banks, such as species composition, seed abundance, and longevity, in order to understand the formation of seed banks and the dynamics of plant communities following disturbances. It clearly demonstrated that the composition of the seed bank depends not only on the composition and production of the present and previous plant communities, but also on the longevity of the seeds of each species under local conditions. This relationship between the composition of the seed bank and the vegetation is particularly important for the vegetation that appears under different management regimes. If Decreaser species cannot seed for any reason during four consecutive years (representing a threshold), maybe as consequence of a drought or any other environmental stress, then seeds of Decreaser species could disappear from the seed bank, thus probably inducing changes in the floristic composition of the plant community at the recruitment phase. Although it would probably not induce a change in vegetation composition, as adult perennial grasses would probably compete with seedlings of the new species. A very long drought or severe grazing, more than four consecutive years, would probably be necessary to produce changes in vegetation composition. On the other hand, a potential change in floristic composition may take place if water availability increases, also through the recruitment from the seed bank, as a high rate of species (13 from 27) are in the soil seed bank but not in aboveground vegetation, probably because they are more mesic.
In summary, the degree of species dissimilarity and shifts in functional groups in the seed bank also provide a good estimate of how far the systems have diverged, and indicate the potential of seed reserves to restore vegetation. Thus, differences in the soil seed bank are likely to reflect manifested properties rather than short-term changes.

\section{LITERATURE CITED}

Adams, K. M. 1996. Influence of sward defoliation and soil disturbance on seedling emergence and survival in the Southern Tall Grassveld. African Journal of Range and Forage Science 13:131-136.

Amaha Kassahun, H., A. Snyman, and G. N. Smit. 2009. Soil seed bank evaluation along a degradation gradient in arid rangelands of the Somali region, eastern Ethiopia. Agricultural, Ecosystems and Environment 129:428-436.

Auffret, A. G., And S. A. 0. Cousins. 2001. Past and present management influences the seed bank and seed rain in a rural landscape mosaic. Journal of Applied Ecology 48:1278-1285.

BAKKER, J. 1989. Nature management by grazing and cutting. Dordrecht, the Netherlands: Kluwer Academic. $316 \mathrm{p}$.

Bekker, R. M., G. L. Verweis, R. E. N. Smith, R. Reine, J. P. Bakker, and S. Schneider. 1997. Soil seed banks in European grasslands: does land use affect regeneration perspective. Journal of Applied Ecology 34:1293-1310.

Bredenkamp, G., and N. van Rooyen. 1996. Dry Sandy Highveld Grassland. In: A. B. Low and A. Rebelo [EDS.]. Vegetation of South Africa, Lesotho and Swaziland. Pretoria, South Africa: Department of Environmental Affairs and Tourism. p. 215-257.

Briske, D. D., T. B. Bestelmeyer, T. K. Stringham, and P. L. Shaver. 2008. Recommendations for development of resilience-based State-and-Transition models. Rangeland Ecology \& Management 61:359-367.

Bullock, J. M., B. Clear-Hill, M. P. Dale, and J. Silvertown. 1994. An experimental study of the effects of sheep grazing on vegetation change in a species-poor grassland and the role of seedling recruitment into gaps. Journal of Applied Ecology 31:193-507.

BusBy, F. E., AND G. F. GifFord. 1981. Effects of livestock grazing on infiltration and erosion rates measured on chained and unchained pinon-juniper sites in southeastern Utah. Journal of Range Management 34:400-405.

Cabellero, I., J. M. Olano, J. Loid, and A. Escudero. 2008. A model for small-scale seed bank and standing vegetation connection along time. Oikos 117:17881795.

Chang, E. R., R. L. Jefferies, and T. J. Carleton. 2001. Relationship between vegetation and soil seed banks in an arctic coastal marsh. Journal of Ecology 89:367-384.

Coluins, S. L., A. K. Knapp, J. M. Briggs, J. M. Blair, and E. M. Steinauer. 1998. Modulation of diversity by grazing and mowing in native tallgrass prairie. Science 280:745-747.

de Villiers, A. J., M. W. van Rooyen, and G. K. Theron. 2003. Similarity between the soil seed bank and the standing vegetation in the Strandveld Succulent Karoo, South Africa. Land Degradation and Development 14:527-540.

DreBer, N. 2011. How best to quantify soil seed banks in arid rangelands of the Nama Karoo? Environmental Monitoring and Assessment 173:813-824.

Dreber, N., And K. J. Esler. 2011. Spatio-temporal variation in soil seed banks under contrasting grazing regimes following low and high seasonal rainfall in arid Namibia. Journal of Arid Environments 75:174-184.

Dreber, N., J. Oldeland, and G. M. W. van Rooyen. 2011. Species, functional groups and community structure in seed banks of the arid Nama Karoo: grazing impacts and implications for rangeland restoration. Agriculture, Ecosystems and Environment 141:399-409.

DU Tolt, T., AND D. AlaRD. 1995. Permanent seed banks in chalk grassland under various management regimes: their role in the restoration of species-rich plant communities. Biodiversity Conservation 4:939-950.

du Tolt, G. van N., H. A. Snyman, and P. J. Malan. 2008. Physical impact of grazing by sheep in the Nama Karoo subshrub/grass rangeland of South Africa on litter and dung distribution. South African Journal of Animal Science 38:326-330.

Edwards, G. R., and M. J. Crawley. 1999. Herbivores, seed banks and seedling recruitment in mesic grassland. Journal of Ecology 87:423-435. 
EverSON, C. S. 1999. Veld burning in different vegetation types. In: N. M. Tainton [ED.]. Veld management in South Africa. Pietermaritzburg, South Africa: University of Natal Press. p. 228-235.

Everson, T. M., R. I. Yeaton, and C. S. Everson. 2009. Seed dynamics of Themeda triandra in the Montana grasslands of South Africa. African Journal of Range and Forage Science 29:19-26.

Fenner, M. 1985. Seed ecology. New York, NY, USA: Chapman and Hall. 144 p.

Fenner, M., And K. Thompson. 2005. The ecology of seeds. Melbourne, VIC, Australia: Cambridge University Press. $215 p$.

FISHER, R. A. 1949. The design of experiments. Edinburg, UK: Coliner and Boyd Ltd. $248 p$.

Froud-Williams, R. J., R. J. Chancellor, and D. S. H. Drennan. 1984. The effects of seed burial and soil disturbance on emergence and survival of arable weeds in relation to minimal cultivation. Journal of Applied Ecology 21:629-641.

Funes, G., S. Basconcelo, S. Dias, and M. Cabido. 1999. Seed size and shape are good predictors of seed persistent in soil in temperate mountain grassland of Argentina. Seed Science Research 9:341-345.

Hardy, M. B., C. R. HuRT, ANd O. J. H. Bosch. 1999. Veld condition assessment. In: N. M. Tainton [ED.]. Veld management in South Africa. Pietermaritzburg, South Africa: University of Natal Press. p. 194-206.

Harrington, G. N., A. D. WiLson, and M. D. Young. 1984. Management of rangeland ecosystems. In: G. N. Harington, A. D. Wilson, and M. D. Young [EDs.]. Management of Australia's rangelands. Melbourne, VIC, Australia: CSIRO Publishing. $277 \mathrm{p}$.

Heitschmidt, R., AND J. Waller. 1983. Short-duration grazing and the Savory Grazing Method in perspective. Rangelands 5:147-150.

HiLL, M. O., D. F. EvenS, AND S. A. BeLL. 1992. Long-term effects of excluding sheep from hill pastures in Wales. Journal of Ecology 80:1-14.

HINTZE, J. L. 1997. Number cruncher statistical system. NCSS: Kaysville, UT, USA.

Hodgkinson, K., and D. Freuderberger. 1997. Production pulses and flow-ons in rangeland landscapes. In: J. Ludwig, D. Tongway, D. Freudenberger, J. Noble, and K. Hodgkinson [EDS.]. Landscape ecology, function and management: principles from Australia's rangelands. Collingwood, VIC, Australia: CSIRO Publishing. p. 110-177.

[ISCW] Institute for Soll, Climate and Water. 2008. ISCW data bank. Pretoria, South Africa: Agricultural Research Council.

Jacquemyn, H., C. van Mechelen, R. Brys, and 0. Honnay. 2001. Management effect on the vegetation and soil seed bank of calcareous grassland: a 11-year experiment. Biological Conservation 144:416-422.

Jensen, H. A. 1969. Content of buried seed in arable soil in Denmark and its relation to weed population. Danske Botaniske Arkiv 27:1-55.

JoNES, R. M. 1968. Seed production of species in the Highveld secondary succession. Journal of Ecology 56:661-666.

Jutlla, H. M., and J. B. Grace. 2002. Effects of disturbance on germination and seedling establishment in a coastal prairie grassland: a test of the competitive release hypothesis. Journal of Ecology 99:291-302.

KInLOCH, J. E., AND M. H. Friedel. 2005. Soil seed reserves in arid grazing lands of central Australia. Part 1: seed bank and vegetation dynamics. Journal of Arid Environments 60:133-161.

Kinucan, R. J., And F. E. Smeins. 1992. Soil seed bank of a semi-arid Texas Grassland under three long-standing (36-years) grazing regimes. American Midland Naturalist 128:11-21.

Koch, M. A., C. Scheriau, M. Schupfner, and K. G. Bernardt. 2011. Long-term monitoring of the restoration and development of limestone grasslands in north western Germany: vegetation screening and soil seed bank analysis. Flora 206:52-65.

Lavorel, S., J. D. Lebreton, J. Debussche, and J. Lepart. 1991. Nested spatial patterns in seed bank and vegetation of Mediterranean oil-fields. Journal of Vegetation Science 2:367-376.

LemeniH, M., AND D. Teketay. 2006. Changes in soil seed bank composition and density following deforestation and subsequent cultivation of a tropical dry Afromontane forest on Ethiopia. Tropical Ecology 47:1-12.

Lererra, P., E. Z. Ortega, M. D. C. Ochoa, 0. R. Vignolio, and 0. N. Fernàndez. 2006. Interactive influences of fire intensity and vertical distribution of seed banks in post-fire recolonization of a tall-tussock grassland in Argentina. Austral Ecology 31:608-622.
Levassor, C., M. Ortega, and B. Peco. 1991. Seed bank dynamics of Mediterranean pastures subjected to mechanical disturbance. Journal of Vegetation Science 1:339-344.

Lòpez-Mariño, A., E. Luis-Calabuig, F. Fillat, and F. F. Bermúdez. 2000. Floristic composition of established vegetation and soil seed bank in pasture communities under different traditional management regimes. Agriculture, Ecosystems and Environment 78:273-282.

Luzuriaga, A. L., A. Escudero, J. M. Olano, and J. Loido. 2007. Regenerative of seed banks following an intense soil disturbance. Acta Oecologica 27:57-66.

MA, M. J., X. H. ZноU, AND G. Z. Du. 2010. Role of soil seed bank along a disturbance gradient in an alpine meadow on the Tibet plateau. Flora 205:128-134.

Mucina, L., And M. C. Rutherford. 2006. The vegetation of South Africa, Lesotho and Swaziland. Strelitzia 19. Pretoria, South Africa: South African National Biodiversity Institute. $808 \mathrm{p}$.

Mueller-Dombols, D., and H. Ellenberg. 1974. Aims and methods of vegetation Ecology. New York, NY, USA: Wiley. $314 \mathrm{p}$.

O'Connor, T. G. 1997. Microsite influence on seed longevity and seedling emergence of a bunch-grass (Themeda triandra) in a semi-arid savanna. African Journal of Range and Forage Science 14:7-11.

O'Connor, T. G., P. Kuyler, K. P. Kirkman, and B. Corcoran. 2010. Which grazing management practices are most appropriate for maintaining biodiversity in South African grassland? African Journal of Range and Forage Science 27:67-76.

O'Connor, T. G., AND G. A. Pickett. 1992. The influence of grazing on seed production and seed banks of some African savanna grasslands. Journal of Applied Ecology 29:247-260.

Olando, J. M., I. Caballero, I. Loidi, and A. Escudero. 2005. Prediction of plant cover from seed bank analysis in a semi-arid plant community on gypsum. Journal of Vegetation Science 16:215-222.

OLFF, H., AND M. E. RitchiE. 1998. Effects of herbivores on grassland plant diversity. Trends in Ecology and Evolution 13:261-265.

Page, M. J., G. S. Baxter, And A. T. Lisle. 2006. Evaluating the adequacy of sampling germinable soil seed banks in semi-arid systems. Journal of Arid Environments 64:323-341.

Page, M. J., And R. J. S. Beeton. 2000. Is the removal of domestic stock sufficient to restore semi-arid conservation areas? Pacific Conservation Biology 6:245-253.

Page, M. J., And R. A. HarRington. 2009. Fourteen months of seed rain in three Australian semi-arid communities. Austral Ecology 34:294-305.

Peco, B., M. Ortego, and C. Levassor. 1998. Similarity between seed bank and vegetation in Mediterranean grassland: a predictive model. Journal of Vegetation Science 9:815-828.

Rusch, G., and J. M. Fernández-Palacios. 1995. The influence of spatial heterogevity of regeneration by seed in a limestone grassland. Journal of Vegetation Science 6:417-462.

Rutherford, M. C., AND L. W. Powrie. 2011. Can heavy grazing on communal land elevate plant species richness levels in the Grassland Biome of South Africa? Plant Ecology 212:1407-1418.

SAS. 2001. Statistical Analysis System user's guide (version 8.1). Cary, NC, USA SAS Institute. $1028 \mathrm{p.}$

Savory, A. 1999. Holistic management. Covelo, CA, USA: Inland Press. 616 p.

SAVory, A., AND S. D. PARSONS. 1980. The Savory grazing method. Rangelands 2:234-237.

Shaukat, S. S., AND I. A. SidDiQul. 2004. Spatial pattern analysis of seeds of an arable soil seed bank and its relationship with above ground vegetation in an arid region. Journal of Arid Environments 57:311-327.

Snyman, H. A. 2000. Soil-water utilisation and sustainability in a semi-arid grassland. Water South Africa 26:331-341.

Snyman, H. A. 2003. Revegetation of bare patches in a semi-arid rangeland of South Africa: an evaluation of various techniques. Journal of Arid Environments $55: 417-432$

Snyman, H. A. 2004. Soil seed bank evaluation and seedling establishment along a degradation gradient in a semi-arid rangeland. African Journal of Range and Forage Science 21:37-47.

Snyman, H. A. 2005. The effect of fire on the soil seed bank of a semi-arid grassland in South Africa. South African Journal of Botany 71:53-60.

Snyman, H. A. 2006. Short-term response of burnt grassland to defoliation in a semiarid climate of South Africa. Journal of Range and Forage Science 23:1-11.

Snyman, H. A. 2009. Root studies on grass species in a semi-arid South Africa along a degradation gradient. Agriculture, Ecosystems and Environment 130:100-108. 
Snyman, H. A. 2010. Longevity of grass seeds in a semi-arid grassland. Grassroots 10(2):8-15.

Snyman, H. A., and H. J. Fouché. 1991. Production and water-use efficiency of semiarid grassland of South Africa as affected by veld condition and rainfall. Water South Africa 17:263-268.

Soll Classification Working Group. 1991. Soil classification: a taxonomic system for South Africa. Pretoria, South Africa: Department of Agriculture Development. $262 \mathrm{p}$.

Solomon, T. B., H. A. Snyman, And G. N. Smit. 2006. Soil seed bank characteristics in relation to land use systems and distance from water in a semi-arid rangeland of southern Ethiopia. South African Journal of Botany 72:263-271.

Thompson, K., S. R. Band, And J. G. Hodgson. 1993. Seed size and shape predict persistence in soil. Functional Ecology 9:236-241.

Thompson, K., And J. P. GRIMe. 1997. Seasonal variation in seed banks of herbaceous species in ten contrasting habitats. Journal of Ecology 67:893-921.

Troltope, W. S. W. 1999. Fire behaviour. In: N. M. Tainton [ED.]. Veld management in South Africa. Pietermaritzburg, South Africa: University of Natal Press. p. 217-228.

Trollope, W. S. W., and A. L. F. Potgieter. 1985. Estimating grass fuel loads with a disc pasture meter in the Kruger National park. Journal of the Grassland Society of South Africa 3:148-152.

van der Westhuizen, H. C., W. L. J. van Rensburg, and H. A. Snyman. 1999. The quantification of rangeland condition in a semi-arid grassland of South Africa. African Journal of Range and Forage Science 16:49-61.
Venable, D. L., and J. S. Brown. 1988. The selective interactions of dispersal, dominancy and seed size as adaptations for reducing risk in variable environments. American Natura 131:360-384.

WaLKER, B. H. 1970. An evaluation of eight methods of botanical analyses on grasslands in Rhodesia. Journal of Applied Ecology 7:403-416.

WaSSIE, A., AND D. TeKETAY. 2006. Soil seed banks in church forests of northern Ethiopia: Implications for the conservations of woody plants. Flora 201:32-43.

WILCOX, B. P., AND M. K. Wood. 1988. Hydrologic impacts of sheep grazing on steep slopes in semi-arid rangeland. Journal of Range Management 41:303-306.

WINER, B. J. 1974. Statistical principles in experimental design. London, UK: McGrawHill. $218 p$.

Wolfson, M. M., And N. M. Tainton. 1999. The morphology and physiology of the major forage plants. In: N. M. Tainton [ED.]. Veld management in South Africa. Scottsville, South Africa: University of Natal Press. p. 54-76.

Yu, S., D. Bell, M. Sternderkg, and P. Kutiel. 2008. The effect of microhabits on vegetation and its relationships with seedlings and soil seed bank in Mediterranean coastal sand dune community. Journal of Arid Environments 72:2040-2053.

Zacharias, P. J. K., C. S. Everson, and M. Walters. 1998. Holistic management (a report-back on a presentation by Allen Savory). Bulletin of the Grassland Society of Southern Africa 8(2):1-5. 